\title{
Agreement between hospital and primary care on diagnostic labeling for COPD and heart failure in Toronto, Canada: a cross-sectional observational study
}

Michelle Greiver ${ }^{1,2,3,4}$, Frank Sullivan ${ }^{1,4,5,6}$, Sumeet Kalia ${ }^{1}$, Babak Aliarzadeh ${ }^{1}$, Deepak Sharma ${ }^{6}$, Steven Bernard ${ }^{6}$, Christopher Meaney $^{1}$, Rahim Moineddin ${ }^{1,4}$, David Eisen ${ }^{1,2,3}$, Navid Rahman ${ }^{6}$ and Tony D'Urzo ${ }^{1}$

Patients with chronic obstructive pulmonary disease (COPD) or heart failure (HF) are frequently cared for in hospital and in primary care settings. We studied labeling agreement for COPD and HF for patients seen in both settings in Toronto, Canada. This was a retrospective observational study using linked hospital-primary care electronic data from 70 family physicians. Patients were 20 years of age or more and had at least one visit in both settings between 1 January 2012 and 31 December 2014. We recorded labeling concordance and associations with clinical factors. We used capture-recapture models to estimate the size of the populations. COPD concordance was 34\%; the odds ratios (ORs) of concordance increased with aging (OR 1.84 for age $75+$ vs. $<65$, $95 \% \mathrm{Cl} 0.92-3.69$ ) and more inpatient admissions (OR 2.89 for $3+$ visits vs. 0 visits, $95 \% \mathrm{Cl} 1.59-5.26$ ). HF concordance was $33 \%$; the ORs of concordance decreased with aging (OR 0.39 for $75+$ vs. $<65,95 \% \mathrm{Cl} 0.18-0.86)$ and increased with more admissions (OR $=$ 2.39; $95 \% \mathrm{Cl} 1.33-4.30$ for $3+$ visits vs. 0 visits). Based on capture-recapture models, $21-24 \%$ additional patients with COPD and $18-20 \%$ additional patients with HF did not have a label in either setting. The primary care prevalence was estimated as 748 COPD patients and $834 \mathrm{HF}$ patients per 100,000 enrolled adult patients. Agreement levels for COPD and HF were low and labeling was incomplete. Further research is needed to improve labeling for these conditions.

npj Primary Care Respiratory Medicine (2018)28:9; doi:10.1038/s41533-018-0076-8

\section{INTRODUCTION}

A foundational activity for the assessment of quality of care is correctly labeling patients that have a health condition of interest so that patient cohorts can be generated. ${ }^{1}$ Quality of care can then be measured, monitored, and improved for this cohort. Patients with high-risk conditions such as heart failure (HF) or chronic obstructive pulmonary disease (COPD) often receive care in both primary care and hospital settings. Exacerbations of COPD and HF are leading causes of hospital admissions in many parts of the world ${ }^{2-4}$ and there is increasing focus on strategies to reverse these trends. Upon discharge from hospital these patients are often advised to follow-up with their primary care physician or health care provider. Understanding how patient diagnoses are documented in these different settings has important clinical and research implications. For example, estimating levels of agreement between diagnostic labeling in primary care practices and hospitals that share the care of patients may facilitate patient management and provide clarity on the state of research preparedness of respective databases, including the size of the populations of interest.

Presently, integrated care systems do not exist in Canada's largest province, Ontario. However, the province has geographically based referral patterns and informal physician networks. ${ }^{5}$ That is, primary care physicians have high "loyalty" and tend to refer to local colleagues and local hospitals. This loyalty has recently been estimated to be about $70 \%,{ }^{5}$ suggesting that most of the clinical data on both primary and inpatient care for majority of patients exists in combined local systems.

In Toronto, Canada, the North York General Hospital (NYGH) and its affiliated primary care physician community have recently cocreated a hospital primary care analytical database, the Health Databank Collaborative (HDC). ${ }^{6}$ This database contains linked clinical data from NYGH and data from the primary care Electronic Medical Records (EMRs) of consenting family physicians, extracted and managed through work done by the UTOPIAN Primary Care Research Network, 1 of 11 networks participating in the Canadian Primary Care Sentinel Surveillance Network, CPCSSN. ${ }^{7}$

Our objectives were to generate cohorts of patients seen in both settings labeled as having $\mathrm{HF}$ or COPD, to estimate agreement on recorded labels of COPD or HF, to determine patient factors associated with agreement on labeling, and to estimate the size of the populations of interest.

\section{RESULTS}

Seventy primary care physicians affiliated with NYGH contributed EMR data to HDC during this study. There were 101,501 patients age 20 or more as of 31 December 2014 enrolled with the practices of these physicians. Patient characteristics are shown in Tables $1 \mathrm{a}$ and $1 \mathrm{~b}$. The odds ratios and adjusted probabilities of

\footnotetext{
${ }^{1}$ Department of Family and Community Medicine, Faculty of Medicine, University of Toronto, Toronto, Canada; ${ }^{2}$ North York Family Health Team, North York, Canada; ${ }^{3}$ Departmen of Family and Community Medicine, North York General Hospital, North York, Canada; ${ }^{4}$ Institute for Clinical Evaluative Sciences, Toronto, Canada; ${ }^{5}$ Medical School, University of St Andrews, St Andrews, UK and ${ }^{6}$ North York General Hospital, North York, Canada Correspondence: Michelle Greiver (mgreiver@rogers.com)
}

Received: 11 October 2017 Revised: 22 January 2018 Accepted: 8 February 2018 Published online: 09 March 2018 
Table 1a. Patient characteristics according to setting with chronic obstructive pulmonary disease labeling

\begin{tabular}{|c|c|c|c|c|c|c|c|}
\hline & \multicolumn{2}{|c|}{$\begin{array}{l}\text { Patients labeled only in primary } \\
\text { care }\end{array}$} & \multicolumn{2}{|c|}{$\begin{array}{l}\text { Patients labeled only in } \\
\text { hospital }\end{array}$} & \multicolumn{2}{|c|}{$\begin{array}{l}\text { Patients labeled in both } \\
\text { settings }\end{array}$} & \multirow{2}{*}{$\begin{array}{l}\text { Total } \\
N\end{array}$} \\
\hline & $N$ & Row percentage (\%) & $N$ & Row percentage (\%) & $N$ & Row percentage (\%) & \\
\hline$<65$ & 52 & 62.7 & 17 & 20.5 & 14 & 16.9 & 83 \\
\hline $65-74$ & 38 & 40.9 & 28 & 30.1 & 27 & 29.0 & 93 \\
\hline No & 200 & 41.4 & 136 & 28.2 & 147 & 30.4 & 483 \\
\hline Yes & 12 & 12.6 & 35 & 36.8 & 48 & 50.5 & 95 \\
\hline \multicolumn{8}{|l|}{ Gender } \\
\hline $\mathrm{F}$ & 125 & 36.7 & 102 & 29.9 & 114 & 33.4 & 341 \\
\hline M & 87 & 36.7 & 69 & 29.1 & 81 & 34.2 & 237 \\
\hline \multicolumn{8}{|l|}{ Income quintiles } \\
\hline 1 (lowest income) & 18 & 33.3 & 8 & 14.8 & 28 & 51.9 & 54 \\
\hline 2 & 25 & 43.1 & 14 & 24.1 & 19 & 32.8 & 58 \\
\hline 3 & 28 & 35.0 & 23 & 28.8 & 29 & 36.3 & 80 \\
\hline 4 & 54 & 46.6 & 28 & 24.1 & 34 & 29.3 & 116 \\
\hline 5 (highest income) & 60 & 39.7 & 34 & 22.5 & 57 & 37.7 & 151 \\
\hline Missing & 27 & 22.7 & 64 & 53.8 & 28 & 23.5 & 119 \\
\hline \multicolumn{8}{|c|}{ No. of visits to emergency department ${ }^{a}$} \\
\hline 0 & 29 & 21.8 & 59 & 44.4 & 45 & 33.8 & 133 \\
\hline 1 & 103 & 49.0 & 49 & 23.3 & 58 & 27.6 & 210 \\
\hline 0 & 132 & 61.4 & 36 & 16.7 & 47 & 21.9 & 215 \\
\hline 1 & 55 & 30.7 & 59 & 33.0 & 65 & 36.3 & 179 \\
\hline 2 & 12 & 14.8 & 35 & 43.2 & 34 & 42.0 & 81 \\
\hline $3+$ & 13 & 12.6 & 41 & 39.8 & 49 & 47.6 & 103 \\
\hline Total & 212 & 36.7 & 171 & 29.6 & 195 & 33.7 & 578 \\
\hline
\end{tabular}

having a concordant diagnostic label for COPD and HF are shown in Tables 2 and 3, respectively.

\section{COPD}

Five hundred seventy-eight patients had charts labeled with a diagnosis of COPD in at least one setting. 59\% were women; the mean age was 77.6 years and the mean number of co-morbidities was $2.49 .36 \%$ of these patients were labeled as having COPD only in primary care, $30 \%$ were labeled only in the hospital and $34 \%$ were concordant (the two settings agreed on labeling). Of those concordant, 128 (65\%) were initially labeled as having a diagnosis of COPD in primary care and 67 (35\%) were initially labeled with COPD in the hospital.
The odds ratios and adjusted probabilities of concordant labeling were higher with increasing age (OR 1.84 for patients age 75 years or more compared to $<65,95 \% \mathrm{Cl} 0.92-3.69$ ), greater number of co-morbidities (OR 4.6 for 3 or more co-morbidities compared to 0 or $1,95 \% \mathrm{Cl} 2.4-8.8)$, and higher number of inpatient admissions (OR 2.9 for 3 or more inpatient admissions compared to none, $95 \% \mathrm{Cl} 1.6-5.3)$. The most socioeconomically deprived patients were more likely to have concordant labeling, although this effect was not statistically significant (OR 0.50 for least deprived vs. most deprived, $95 \% \mathrm{Cl} 0.25-1.02$ ). There were no significant differences by patient gender, number of emergency department visits, or number of primary care encounters.

$37 \%$ of patients with COPD were seen in the ED only (no inpatient admissions). $21.9 \%$ of those had concordant labeling as shown in Tables $1 \mathrm{a}$ and $1 \mathrm{~b}$. The adjusted probability of 
Table 1b. Patient characteristics according to setting with heart failure labeling

\begin{tabular}{|c|c|c|c|c|c|c|c|}
\hline & \multicolumn{2}{|c|}{$\begin{array}{l}\text { Patients labeled only in } \\
\text { primary care }\end{array}$} & \multicolumn{2}{|c|}{$\begin{array}{l}\text { Patients labeled only in } \\
\text { hospital }\end{array}$} & \multicolumn{2}{|c|}{$\begin{array}{l}\text { Patients labeled in both } \\
\text { settings }\end{array}$} & \multirow{2}{*}{$\begin{array}{l}\text { Total } \\
N\end{array}$} \\
\hline & $N$ & Percentage (\%) & $N$ & Percentage (\%) & $N$ & Percentage (\%) & \\
\hline$<65$ & 8 & 21.1 & 13 & 34.2 & 17 & 44.7 & 38 \\
\hline $65-74$ & 21 & 28.4 & 25 & 33.8 & 28 & 37.8 & 74 \\
\hline No & 113 & 21.4 & 256 & 48.5 & 159 & 30.1 & 528 \\
\hline Yes & 20 & 12.3 & 77 & 47.2 & 66 & 40.5 & 163 \\
\hline \multicolumn{8}{|l|}{ Gender } \\
\hline $\mathrm{F}$ & 83 & 19.3 & 217 & 50.5 & 130 & 30.2 & 430 \\
\hline M & 50 & 19.2 & 116 & 44.4 & 95 & 36.4 & 261 \\
\hline \multicolumn{8}{|l|}{ Income quintiles } \\
\hline 1 (lowest income) & 13 & 21.0 & 23 & 37.1 & 26 & 41.9 & 62 \\
\hline 2 & 15 & 20.8 & 25 & 34.7 & 32 & 44.4 & 72 \\
\hline 3 & 25 & 27.2 & 32 & 34.8 & 35 & 38.0 & 92 \\
\hline 4 & 27 & 22.1 & 53 & 43.4 & 42 & 34.4 & 122 \\
\hline 5 (highest income) & 34 & 21.7 & 57 & 36.3 & 66 & 42.0 & 157 \\
\hline Missing & 19 & 10.2 & 143 & 76.9 & 24 & 12.9 & 186 \\
\hline \multicolumn{8}{|c|}{ No. of visits to emergency department ${ }^{\mathrm{a}}$} \\
\hline 0 & 21 & 9.7 & 121 & 55.8 & 75 & 34.6 & 217 \\
\hline 1 & 57 & 28.4 & 86 & 42.8 & 58 & 28.9 & 201 \\
\hline \multicolumn{8}{|c|}{ No. of encounters with primary care physician ${ }^{a}$} \\
\hline \multicolumn{8}{|c|}{ No. of inpatient admissions ${ }^{a}$} \\
\hline 0 & 68 & 48.9 & 36 & 25.9 & 35 & 25.2 & 139 \\
\hline 1 & 46 & 18.1 & 122 & 48.0 & 86 & 33.9 & 254 \\
\hline 2 & 10 & 7.9 & 73 & 57.9 & 43 & 34.1 & 126 \\
\hline $3+$ & 9 & 5.2 & 102 & 59.3 & 61 & 35.5 & 172 \\
\hline Total & 133 & 19.2 & 333 & 48.2 & 225 & 32.6 & 691 \\
\hline
\end{tabular}

concordance for patients with ED visits only was $25 \%(95 \% \mathrm{Cl}$ 16-37\%) as shown in Table 2.

\section{Heart failure}

Six hundred ninty-one patients had charts labeled with a diagnosis of HF in at least one setting. $62 \%$ were women; the mean age was 83 years and the mean number of co-morbidities was $2.0 .19 \%$ were labeled as having HF only in primary care, $48 \%$ were labeled only in the hospital and $33 \%$ were concordant (the two settings agreed on labeling). Of those concordant, 88 (39\%) were initially labeled as having a diagnosis of HF in primary care and 137 (61\%) were initially labeled in the hospital.

The odds ratios and adjusted probabilities of patients having a label of HF in both settings decreased as age increased (OR 0.39 for patients age 75 years or more compared to $<65,95 \% \mathrm{Cl}$ $0.18-0.86)$. Patients that died during the period of observation were more likely to have agreement on diagnostic labeling $(\mathrm{OR}=$ $1.99,95 \% \mathrm{Cl} 1.29-3.08)$. An increasing number of co-morbidities also increased the likelihood of concordance $(\mathrm{OR}=2.04$ for 3 or more co-morbidities vs. none; $95 \% \mathrm{Cl} 1.26-3.09$ ), as were more inpatient visits $(\mathrm{OR}=2.39 ; 95 \% \mathrm{Cl} 1.33-4.30$ for $3+$ visits vs. 0 visits). There were no significant differences by patient gender, income quintiles, number of emergency department visits, or number of primary care encounters.

$20 \%$ of patients with HF were seen in the ED only (no inpatient admissions); $25.2 \%$ of those had concordant labeling as shown in Tables $1 \mathrm{a}$ and $1 \mathrm{~b}$. The adjusted probability of concordance for patients with ED visits only was $32 \%$ (95\% Cl $20-46 \%)$, as shown in Table 2. 
Table 2. Odds ratios of having concordant labeling (label present in both settings) for COPD and heart failure

\begin{tabular}{|c|c|c|c|c|}
\hline Effect & Index group & Reference group & $\begin{array}{l}\text { COPD } \\
\text { Odds ratio ( } 95 \% \text { confidence interval) }\end{array}$ & $\begin{array}{l}\text { Heart failure } \\
\text { Odds ratio ( } 95 \% \text { confidence interval) }\end{array}$ \\
\hline Age range (years) & $65-74$ & $<65$ & $1.37(0.62-3.05)$ & $0.47(0.19-1.17)$ \\
\hline Gender & $\mathrm{F}$ & $\mathrm{M}$ & $0.92(0.62-1.38)$ & $0.72(0.49-1.05)$ \\
\hline Deceased & Yes & No & $1.9(1.12-3.23)$ & $1.99(1.29-3.08)$ \\
\hline \multirow[t]{4}{*}{ Income quintiles } & 2 & 1 & $0.35(0.15-0.82)$ & $1.33(0.62-2.86)$ \\
\hline & 3 & 1 & $0.51(0.23-1.11)$ & $1.07(0.52-2.21)$ \\
\hline & 4 & 1 & $0.35(0.17-0.74)$ & $0.73(0.37-1.47)$ \\
\hline & 5 & 1 & $0.5(0.25-1.02)$ & $1.13(0.58-2.21)$ \\
\hline No. of ED visits ${ }^{a}$ & 1 & 0 & $1.05(0.58-1.9)$ & $0.68(0.41-1.15)$ \\
\hline \multirow{2}{*}{ No. of inpatient visits ${ }^{a}$} & 2 & 0 & $2.47(1.29-4.73)$ & $2.05(1.08-3.89)$ \\
\hline & $3+$ & 0 & 2.89 (1.59-5.26) & $2.39(1.33-4.3)$ \\
\hline \multirow[t]{2}{*}{ No. of primary care visits ${ }^{a}$} & 3 to 9 & 1 to 2 & $0.84(0.26-2.67)$ & $1.02(0.42-2.47)$ \\
\hline & $10+$ & 1 to 2 & $0.58(0.19-1.72)$ & $1.23(0.54-2.8)$ \\
\hline
\end{tabular}

\section{Capture-recapture models}

Using capture-recapture models, we determined that $21-24 \%$ additional patients with COPD and $18-20 \%$ additional patients with HF did not have the condition labeled in their chart in either setting. The data are presented in Tables $4 \mathrm{a}$ and $4 \mathrm{~b}$ and Fig. 1. After adjustment for patient characteristics, the population size was estimated to be 760 patients $(95 \% \mathrm{Cl} 695-837)$ with COPD and 847 patients $(95 \% \mathrm{Cl} 787-918)$ with HF. Given that there were 101,501 patients enrolled to the primary care physicians in this cohort, we expect to find 748 COPD patients per 100,000 enrolled adult patients and $834 \mathrm{HF}$ patients per 100,000 enrolled adult patients.

\section{DISCUSSION}

We found significant disagreement between hospital and primary care records on labeling for COPD and HF as the two settings agreed on only a third of patients. About a fifth of all patients had no diagnostic labels in either setting. Agreement on a diagnostic label was more likely when there were more inpatient admissions but not when there were more primary care visits. The estimated prevalence of COPD or HF associated with hospitalization in primary care practices was slightly less than $1 \%$ for each condition.

There is scant information on labeling agreement between hospital and primary care for chronic conditions with high impact on health; one study in Spain found low concordance for a number of conditions, including COPD and HF. ${ }^{8}$ A study in Scotland found low levels of concordance for incident myocardial infarcts, strokes, and ischemic heart disease. ${ }^{9}$ The lack of agreement on diagnostic labels in our study is surprising, given the fact that the two conditions we studied are associated with significant morbidity and mortality and are leading causes of hospital admission and re-admissions. ${ }^{2-4}$

Misclassifications of COPD and HF (both under-diagnosis and overdiagnosis) are common. ${ }^{10-12}$
Chart audits for the presence of appropriate testing and diagnostic criteria would be needed in both settings to determine diagnostic accuracy of in this study. This would include spirometry for COPD $^{13}$ and echocardiography and brain natriuretic peptide for HF. ${ }^{12,14}$ We therefore make no inferences about the accuracy of labeling. It would be reasonable to assume that there are errors in both settings and this would at least partially explain the lack of agreement.

The prevalence of COPD in Canada has been estimated at 4\% using self-reports, which underestimate COPD. ${ }^{15}$ Recent spirometry testing for a random sample of the population as part of the Canadian Health Measures Survey found a prevalence of $17 \%$ for COPD. ${ }^{16}$ The prevalence of moderate to severe COPD (GOLD III and IV) was $1 \% .{ }^{16}$ We report an estimated prevalence of $0.9 \%$ in this population of patients with hospital admissions. We are not able to determine from our study whether this difference is due to under-diagnosis or to our population having more severe disease. Under-diagnosis of COPD is a common finding in primary care, ${ }^{17,18}$ and this might in part be related to lack of access to spirometry in primary care. $^{19}$

It is relevant to note that even among patients admitted to hospital with a diagnosis of COPD, less than $10 \%$ of such patients undergo confirmatory spirometry and about one-third do not demonstrate spirometric features compatible with COPD. ${ }^{20}$ These data highlight that factors affecting diagnostic labeling may include availability of objective data to confirm COPD diagnosis as well as practices utilized in both the hospital and community clinic settings which drive how data are entered into medical records.

The HF prevalence of $0.8 \%$ in our study is comparable to the self-reported prevalence of $\mathrm{HF}$ in Canada of $1 \%{ }^{21}$ Yearly rates of hospital admission for HF in Canada have been decreasing and were 204 per 100,000 population in 2014. ${ }^{22}$ However, a recent study found that $83.1 \%$ of patients followed with HF in the community have had at least one hospitalization in a 5-year period, with the majority of reasons $(61.9 \%)$ being noncardiovascular. ${ }^{23}$ 
More patients were labeled with COPD only in the community rather than only in the hospital. Many cases of COPD are mild (GOLD I and II) and may not be perceived as impacting morbidity in the hospital but could be labeled in the community due to case finding for patients who smoke or have clinical features such as chronic cough. The opposite was present for HF, with $48 \%$ of patients labeled only in the hospital, even though they were seen by their family physician after a hospitalization. Both COPD and HF are Ambulatory Care Sensitive Conditions, meaning that optimal

Table 3. Adjusted probabilities of having concordant labeling (label present in both settings) for COPD and heart failure

\begin{tabular}{|c|c|c|c|}
\hline Effect & Value & $\begin{array}{l}\text { COPD } \\
\text { Adjusted probability } \\
\text { ( } 95 \% \text { confidence } \\
\text { interval) }\end{array}$ & $\begin{array}{l}\text { Heart failure } \\
\text { Adjusted probability } \\
\text { (95\% confidence } \\
\text { interval) }\end{array}$ \\
\hline \multirow[t]{3}{*}{ Age range (years) } & $<65$ & $0.32(0.18-0.5)$ & $0.59(0.39-0.76)$ \\
\hline & $65-74$ & $0.39(0.25-0.55)$ & $0.40(0.26-0.56)$ \\
\hline & $75+$ & $0.46(0.35-0.57)$ & $0.36(0.27-0.45)$ \\
\hline \multirow[t]{2}{*}{ Gender } & $\mathrm{F}$ & $0.38(0.27-0.5)$ & $0.41(0.3-0.53)$ \\
\hline & M & $0.40(0.28-0.53)$ & $0.49(0.37-0.62)$ \\
\hline \multirow[t]{2}{*}{ Deceased } & Yes & $0.47(0.32-0.62)$ & $0.53(0.4-0.67)$ \\
\hline & No & $0.31(0.22-0.43)$ & $0.37(0.27-0.48)$ \\
\hline \multirow[t]{3}{*}{ No. of co-morbidities } & $0-1$ & $0.21(0.13-0.32)$ & $0.35(0.24-0.47)$ \\
\hline & 2 & $0.44(0.3-0.59)$ & $0.48(0.35-0.62)$ \\
\hline & $3+$ & $0.55(0.41-0.69)$ & $0.52(0.38-0.65)$ \\
\hline \multirow[t]{5}{*}{ Income quintiles } & 1 & $0.58(0.4-0.74)$ & $0.48(0.31-0.65)$ \\
\hline & 2 & $0.32(0.19-0.5)$ & $0.55(0.39-0.7)$ \\
\hline & 3 & $0.41(0.26-0.57)$ & $0.50(0.34-0.65)$ \\
\hline & 4 & $0.32(0.2-0.47)$ & $0.40(0.27-0.55)$ \\
\hline & 5 & $0.41(0.28-0.55)$ & $0.51(0.38-0.65)$ \\
\hline \multirow[t]{4}{*}{ No. of ED visits ${ }^{a}$} & 0 & $0.36(0.24-0.5)$ & $0.49(0.36-0.61)$ \\
\hline & 1 & $0.37(0.24-0.51)$ & $0.39(0.27-0.53)$ \\
\hline & 2 & $0.45(0.29-0.62)$ & $0.52(0.37-0.67)$ \\
\hline & $3+$ & $0.38(0.26-0.52)$ & $0.40(0.26-0.55)$ \\
\hline \multirow{4}{*}{$\begin{array}{l}\text { No. of inpatient } \\
\text { visits }^{\mathrm{a}}\end{array}$} & 0 & $0.25(0.16-0.37)$ & $0.32(0.20-0.46)$ \\
\hline & 1 & $0.39(0.26-0.53)$ & $0.47(0.35-0.6)$ \\
\hline & 2 & $0.45(0.3-0.61)$ & $0.49(0.34-0.64)$ \\
\hline & $3+$ & $0.49(0.33-0.64)$ & $0.53(0.39-0.66)$ \\
\hline \multirow{3}{*}{$\begin{array}{l}\text { No. of primary care } \\
\text { visits }^{\mathrm{a}}\end{array}$} & $1-2$ & $0.45(0.21-0.7)$ & $0.43(0.24-0.64)$ \\
\hline & $3-9$ & $0.40(0.27-0.55)$ & $0.43(0.3-0.58)$ \\
\hline & $10+$ & $0.32(0.24-0.41)$ & $0.48(0.38-0.58)$ \\
\hline
\end{tabular}

COPD chronic obstructive pulmonary disease, $E D$ emergency department

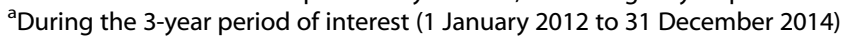

care in the community can reduce hospitalizations and emergency department visits. ${ }^{24}$ Appropriate diagnostic labeling of patient charts is needed to monitor quality of care. ${ }^{1}$ Reasons for lack of labeling in the community following hospitalization could include poor communication between hospital and primary care ${ }^{25}$ or disagreement about the diagnosis. Reducing this gap in concordance is important it terms of promoting a robust primary care data base for research.

Concordance for both conditions increased with an increasing number of hospitalizations during the 3-year period, perhaps reflecting more opportunities for reports mentioning the conditions being forwarded from the hospital to primary care. Older age was associated with greater concordance for COPD but the opposite for HF; this unexpected finding should be confirmed and would benefit from further study. Some factors that might have influenced our findings include the fact that approximately half of hospital re-admissions in patients with $\mathrm{HF}$ are related to comorbidities, polypharmacy, and other conditions associated with $\mathrm{HF}^{26}$

Our patients with HF were older than patients with COPD (77 vs. 83 years). Furthermore, we noted that there were more female patients among our population of HF patients. Older HF patients are more likely to be female. ${ }^{26}$ These patients also tend to have higher rates of non-cardiovascular conditions, ${ }^{26}$ which may drive patients to seek medical attention and influence diagnostic labeling in both the primary and hospital settings.

It is important to mention that we did not evaluate concordance among those patients with a diagnosis of both HF and COPD. Among individuals with $\mathrm{HF}$ the prevalence of COPD ranges between 20 and $32 \%$, and reports suggest that $10 \%$ of hospitalized HF patients also suffer from COPD. ${ }^{27}$ Conversely, HF is prevalent in more than $20 \%$ of patients with COPD. ${ }^{28,29}$ It was beyond the scope of this study to understand how concomitant illness with COPD and HF might influence concordance rates in the two settings we studied.

Understanding the concordance of this particularly high-risk population between the two settings and confirmation of diagnosis by echocardiography and spirometry would be advantageous in the overall care of such individuals.

\section{Limitations}

The study had several strengths. It reflected data from routine clinical care for patients with COPD and HF in community-based primary care and hospital care. Data were extracted from several different EMR platforms, accounting for a variety of EMR-specific data entry processes for diagnostic labeling by clinicians. Despite these strengths, this study includes several shortcomings. This was a convenience sample of primary care practices that contributed EMR data to UTOPIAN, rather than a random sample. Correction of an inaccurate diagnosis is another explanation for some of the discrepancy. For example, a chesty smoker may be coded as

Table 4a. Proportions of patients with a label of COPD captured in different settings, estimated additional proportion of missing patients and estimated population size

\begin{tabular}{llllll}
\hline Model & $\begin{array}{l}\text { Patients labeled } \\
\text { in hospital }\end{array}$ & $\begin{array}{l}\text { Patients labeled in } \\
\text { primary care }\end{array}$ & $\begin{array}{l}\text { Patients labeled } \\
\text { in both }\end{array}$ & $\begin{array}{l}\text { Additional patients not } \\
\text { labeled in either setting }\end{array}$ & $\begin{array}{l}\text { Estimated population size } \\
(95 \% \text { confidence interval) }\end{array}$ \\
\hline COPD $=$ no covariate & $30 \%$ & $37 \%$ & $34 \%$ & $24 \%(19-30 \%)$ & $764(710-827)$ \\
COPD $=$ age & $30 \%$ & $36 \%$ & $34 \%$ & $24 \%(19-30 \%)$ & $763(709-826)$ \\
COPD $=$ age + sex & $32 \%$ & $34 \%$ & $34 \%$ & $24 \%(18-30 \%)$ & $763(708-828)$ \\
COPD $=$ age + sex + income & $29 \%$ & $34 \%$ & $37 \%$ & $21 \%(15-27 \%)$ & $734(681-796)$ \\
quintile & & $34 \%$ & $34 \%$ & $24 \%(17-31 \%)$ & $760(695-837)$ \\
COPD $=$ age + sex + income & $32 \%$ & & & & \\
quintile + comorbidities & & & &
\end{tabular}


Table $\mathbf{4 b}$. Proportions of patients with a label of heart failure captured in different settings, estimated additional proportion of missing patients and estimated population size

\begin{tabular}{llllll}
\hline Model & $\begin{array}{l}\text { Patients labeled } \\
\text { in hospital }\end{array}$ & $\begin{array}{l}\text { Patients labeled in } \\
\text { primary care }\end{array}$ & $\begin{array}{l}\text { Patients labeled } \\
\text { in both }\end{array}$ & $\begin{array}{l}\text { Additional patients not } \\
\text { labeled in either setting }\end{array}$ & $\begin{array}{l}\text { Estimated population size } \\
\text { (95\% confidence interval) }\end{array}$ \\
\hline $\mathrm{HF}=$ no covariate & $46 \%$ & $19 \%$ & $35 \%$ & $20 \%(14-26 \%)$ & $859(799-929)$ \\
$\mathrm{HF}=$ age & $46 \%$ & $19 \%$ & $35 \%$ & $20 \%(14-26 \%)$ & $860(799-930)$ \\
$\mathrm{HF}=$ age + sex & $36 \%$ & $25 \%$ & $36 \%$ & $20 \%(14-27 \%)$ & $867(800-946)$ \\
$\mathrm{HF}=$ age + sex + income & $34 \%$ & $26 \%$ & $39 \%$ & $19 \%(12-25 \%)$ & $849(787-923)$ \\
quintile & & $26 \%$ & $39 \%$ & $18 \%(12-25 \%)$ & $847(787-918)$ \\
HF = age + sex + income & $34 \%$ & & & & \\
quintile + comorbidities & & & &
\end{tabular}

$C O P D$ chronic obstructive pulmonary disease, $H F$ heart failure

(a) COPD

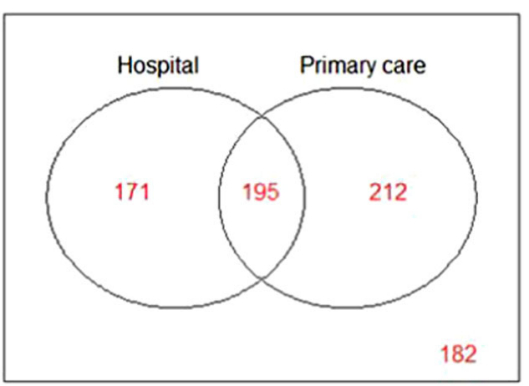

(b) Heart failure

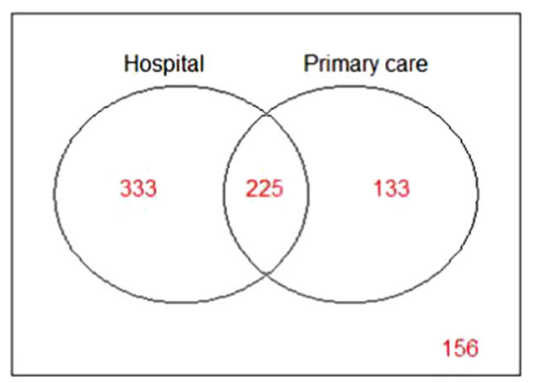

Fig. 1 Venn diagrams for number of patients labeled for COPD and heart failure per site, using capture-recapture models: a COPD, One hundred seventy-one patients were labeled only in the hospital, 212 were labeled only in primary care, and 195 in both. One hundred eightytwo patients were not labeled in either setting. b Heart failure, Three hundred thirty-three patients were labeled only in the hospital, 133 were labeled only in primary care, and 225 in both. One hundred fifty-six patients were not labeled in either setting

"COPD" by a physician in the ED and subsequently corrected by the family physician after spirometry. A breathless patient coded by a family physician as HF may be corrected after testing during a hospital admission. However, we note that in Canadian family practices, adding a health condition to the summary health profile is not a trivial activity, as this is considered the "master list" for significant problems in the health record, and is recognized as such by regulatory authorities. ${ }^{30}$

We only counted hospital admissions and ED visits; there are several other reasons for a visit to the hospital, including diagnostic imaging. We would not necessarily expect complete labeling for major health conditions if the patient presented for imaging only. It is reasonable, however, to expect major conditions, such as COPD or HF, to be labeled in a patient's chart during a hospital admission.

Patients may present to ED with minor conditions; coding for COPD or HF may not have been entered in those cases. We were unable to exclude visits for minor conditions from the dataset and provided results excluding patients seen only in ED. In a random audit of 100 patients with COPD and HF for this study, only three ED visits with COPD and two ED visits with HF were not followed by an admission.

We did not collect several variables relevant to the diagnosis of COPD or HF, as these were not available in the HDC database. These variables include echocardiograms and spirometry results. The data were from a single hospital, and do not reflect information on patients admitted to other hospitals in the area. However, regional loyalty in our setting is reasonably high at an estimated $70 \%$, reflecting the share of patients with COPD or HF from primary care physicians seen at the hospital. ${ }^{5}$ We studied labeling concordance, which would not be impacted by admission elsewhere. All family physicians in this study were affiliated with NYGH.

\section{Conclusions}

We found low rates of labeling agreement for COPD and HF between a hospital and its primary care community as well as missing labels in both settings. This points to opportunities to improve the documentation of these high-risk, high-cost conditions. Further research is needed to understand and explore factors that influence diagnostic labeling and agreement. Identifying strategies to improve diagnostic labeling between the hospital and community clinics may also serve to develop robust data bases which can be used to promote patient care and collaborative research initiatives.

\section{METHODS}

\section{Study design}

This was a cross-sectional retrospective observational study using data from the NYGH HDC database; we applied the STROBE checklist for reporting observational studies. ${ }^{31}$

\section{Data sources}

EMR data routinely entered in primary care charts were used for this study. These data were extracted and managed through work done by UTOPIAN, the University of Toronto Practice-Based Research Network. UTOPIAN is one of 12 networks participating in the Canadian Primary Care Sentinel Surveillance Network, CPCSSN. ${ }^{7}$ CPCSSN is Canada's largest primary care EMR-based research and surveillance database; all contributing networks 
Patients age 20 or over and enrolled to participating

family physician as of

31 December 2014 or date deceased

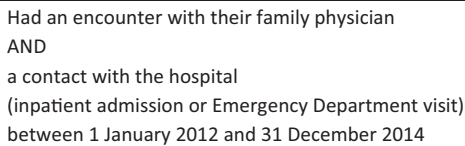

Must have been seen in the other setting

after the label was initially entered in the record in one setting

Fig. 2 Flow diagram for cohort generation, COPD

use similar data extraction and management processes and these have been previously described. ${ }^{7}$ Briefly, consenting family physicians and other primary care providers contribute de-identified EMR data to the UTOPIAN data repository; posters informing patients about the study are present in the waiting rooms of participating practices, patients can opt-out if they choose to do so. ${ }^{7,32}$

The primary care data were linked to the hospital's database using an encrypted identifier for each patient. ${ }^{6}$ The linked data were analyzed.

\section{Patient population}

The population of interest consisted of patients age 20 or more as of 31 December 2014 or as of date deceased, seen at least once in both settings (hospital and primary care) during a 3-year period (1 January 2012 to 31 December 2014). Patients were enrolled to a family physician contributing data to the HDC. There must have been a diagnostic label of COPD or HF recorded in at least one setting at any time prior to 31 December 2014. There must have been at least one visit to the primary care physician following the hospital visit where HF or COPD had initially been recorded, or one Hospital visit where there was a pre-existing HF or COPD diagnosis in the primary care record. The generation of the cohort for COPD is described in Fig. 2. A similar process was used for HF.

For the hospital, standardized information on acute inpatient care and emergency care is extracted from each chart and recorded using ICD10 codes in the Canadian Institute for Health Information Discharge Abstract Database $(\mathrm{CIHI} D A D)^{33}$ and $\mathrm{ClHI}$ National Ambulatory Care Reporting System (NACRS). ${ }^{34}$ Trained auditors abstract data from the chart, using a standardized manual, and record findings as structured data. The CIHI diagnostic types used were the Most Responsible Diagnosis and pre-admit comorbidity. ${ }^{33}$

We used ICD-10-CA codes for HF consistent with those in CIHI DAD/ NACRS and recommended as indicating HF by the recent Health Quality Ontario Quality-Based Procedures: 150.x, 125.5, 140.x, 141.x, 142.x, 143.x. ${ }^{35}$ For COPD, we used ICD-10-CA J41-J44. ${ }^{36}$

For primary care, we used data present in the summative health profile of the chart. In Canada, provincial licensing organizations recommend using the summative health profile as a standard area for recording the presence of important chronic conditions in primary care. ${ }^{30,37}$ We included patients with a coded diagnosis for HF (ICD9 428) or COPD (ICD9 491, 492, 496) or free text indicating either condition. A similar approach was used to validate the CPCSSN case definitions. ${ }^{38}$ Validation studies report good to excellent sensitivity and specificity for ICD9 codes 491, 492, and 496 for $\mathrm{COPD}^{39}$ and 498 for HF. ${ }^{40}$ CPCSSN has a coding tool that is applied when free text for diagnosis is present in the health profile and converts free text to ICD9 codes; we used this coding tool. In summary, any entry containing COPD, CHRONIC BRONCHITIS, EMPHYSEMA, CHRONIC AIRWAY OBSTRUCTION, CHRONIC OBSTRUCTIVE PULMONARY DISEASE, and any other keywords that indicate COPD were coded as COPD. HEART FAILURE, HEARTFAILURE, HT FAILURE, BIVENTRICULAR FAILURE, CARDIAC FAILURE, WEAK HEART, CHF, CONGESTIVE HEART DISEASE, VENTRICULAR FAILURE were used for HF. The tool excludes data where keywords indicate uncertainty about the diagnosis; these include "not", "unlikely", "unremarkable", "absence of", "check" before diagnosis keyword, or "?" before or after the diagnosis keyword.
The following data elements were extracted from the HDC database: patient age as of 31 December 2014 or date deceased, patient gender, presence of co-morbidities, socioeconomic quintiles, and health care utilization (number of primary care visits, hospital utilization). Comorbidities were derived from eight CPCSSN validated disease case definition algorithms: diabetes, hypertension, osteoarthritis, depression, COPD, dementia, epilepsy, Parkinson's disease. ${ }^{38}$ One of the conditions classified was COPD, so seven co-morbidities were measured for patients with COPD.

Hospital utilization was determined by the number of emergency department visits and inpatient admissions. If there was an emergency department visit followed by an inpatient admission, this was counted as one hospital inpatient admission.

We used geographically derived information to calculate income level. ${ }^{41}$ The Postal Code Conversion File, available from Statistics Canada, was used to link the six-character postal codes to the standard 2011 Census dissemination areas. Dissemination areas are small, stable parts of neighborhoods that include between 400 and 700 persons (http:// www12.statcan.gc.ca/census-recensement/2011/ref/dict/geo021-eng.cfm). Subsequently, the Postal Code Conversion File was used to assign neighborhood income. ${ }^{42}$

\section{Statistical analysis}

All statistical analyses were conducted using SAS software, version 9.4 (SAS Institute). We recorded whether labeling for COPD and HF was present in the hospital and primary care records. We examined the proportion of patients with concordant diagnostic labeling (where a label for each condition was present in both settings) and association between labeling concordance and clinical factors using random effects logistic regression model. We also used capture-recapture model to estimate the size of the patient population with COPD or HF that had been looked after in both hospital and primary care. Capture-recapture consists of taking a sample from a population, tagging each item sampled and placing Manuscript, all changes accepted them back. The population is then re-sampled and the tagged items are counted. The proportion of items tagged in the new sample reflects the proportion in the population and the size of the population can then be estimated. More details on capture-recapture model are provided in the Supplementary File.

This study was reviewed and appoved by the Research Ethics Board (REB) at the NYGH. UTOPIAN has received REB approval from the University of Toronto and from the NYGH for the collection of EMR data. All participating primary care providers have provided written informed consent for the collection and analysis of their EMR data.

\section{Data availability}

The data that support the findings of this study are available from the NYGH's Office of Research and Innovations. Requests for data may be made at http://www.nygh.on.ca/Default.aspx?cid=3157\&lang=1. The data are not publicly available as they may contain patient-related information that could compromise patient privacy. Derived data supporting the findings of this study are available from the corresponding author upon request.

\section{ACKNOWLEDGEMENTS}

The authors are grateful to the North York General Foundation for financial support through the Exploration Fund. Dr. Greiver holds an investigator award from the Department of Family and Community Medicine, University of Toronto and was supported by a research stipend from North York General Hospital.

\section{AUTHOR CONTRIBUTIONS}

M.G. and F.S. contributed to conception and design. B.A. was responsible for acquisition of data. S.K., C.M., and R.M. contributed substantially to the analysis of data. T.D. provided content-specific expertise on COPD and HF. M.G. drafted the initial version of the article. All authors contributed to the interpretation of data. All authors reviewed and revised the article for important intellectual content and gave final approval of the version to be published. M.G. is the guarantor of this work and, as such, had full access to all the data in the study and takes responsibility for the integrity of the data and the accuracy of the data analysis. 


\section{ADDITIONAL INFORMATION}

Supplementary information accompanies the paper on the npj Primary Care Respiratory Medicine website (https://doi.org/10.1038/s41533-018-0076-8).

Competing interests: The authors declare no competing financial interests.

Publisher's note: Springer Nature remains neutral with regard to jurisdictional claims in published maps and institutional affiliations.

\section{REFERENCES}

1. Molina-Ortiz, E. I., Vega, A. C. \& Calman, N. S. Patient registries in primary care: essential element for quality improvement. Mt. Sinai J. Med. 79, 475-480 (2012).

2. Government of Canada. Public Health Agency of Canada: leading causes of death and hospitalization in Canada 2010. http://www.phac-aspc.gc.ca/publicat/lcdpcd97/index-eng.php (2016).

3. Pfuntner, A., Wier, L. M. \& Stocks, C. HCUP Statistical Brief 148: Most Frequent Conditions in U.S. Hospitals, 2010. (AHRQ, Rockville, MD, 2013).

4. Hines, A. L., Barrett, M. L., Jiang, H. J. \& Steiner, C. A. HCUP Statistical Brief 172: Conditions with the Largest Number of Adult Hospital Readmissions by Payer, 2011. (AHRQ, Rockville, MD, 2014).

5. Stukel, T. A. et al. Multispecialty physician networks in Ontario. Open Med. 7, e40-e55 (2013).

6. An., J., Keshavjee, K., Mirza, K., Vassanji, K. \& Greiver, M. Bridging a divide: architecture for a joint hospital-primary care data warehouse. Stud. Health Technol. Inform. 208, 45-49 (2015).

7. Birtwhistle, R. et al. Building a pan-Canadian primary care sentinel surveillance network: initial development and moving forward. J. Am. Board Fam. Med. 22, 412-422 (2009).

8. Revilla-Lopez, C., Calderon-Larranaga, A., Enriquez-Martin, N. \& Prados-Torres, A. Low concordance between primary care and hospital clinical information. Aten Primaria 48, 244-250 (2016).

9. Payne, R. A., Abel, G. A. \& Simpson, C. R. A retrospective cohort study assessing patient characteristics and the incidence of cardiovascular disease using linked routine primary and secondary care data. BMJ Open 2, e000723 (2012).

10. Strong, $M$. et al. Accuracy of diagnosis and classification of COPD in primary and specialist nurse-led respiratory care in Rotherham, UK: a cross-sectional study. Prim. Care Respir. J. 23, 67-73 (2014).

11. Skinner, T. R., Scott, I. A. \& Martin, J. H. Diagnostic errors in older patients: a systematic review of incidence and potential causes in seven prevalent diseases. Int. J. Gen. Med. 9, 137-146 (2016).

12. Barents, M. et al. Prevalence and misdiagnosis of chronic heart failure in nursing home residents: the role of B-type natriuretic peptides. Neth. Heart J. 16, 123-128 (2008).

13. Qaseem, A. et al. Diagnosis and management of stable chronic obstructive pulmonary disease: a clinical practice guideline update from the American College of Physicians, American College of Chest Physicians, American Thoracic Society, and European Respiratory Society. Ann. Intern. Med. 155, 179-191 (2011).

14. Government of Canada, Statistics Canada. Diabetes, by age group and sex Ottawa: statistics Canada. http://www.statcan.gc.ca/tables-tableaux/sum-som/ 101/cst01/health53b-eng.htm (updated 7 March 2016) (2013).

15. Canadian Community Health Survey (CCHS). Surveys and statistical programsannual component (CCHS) (2015).

16. Evans, J., Chen, Y., Camp, P. G., Bowie, D. M. \& McRae, L. Estimating the prevalence of COPD in Canada: Reported diagnosis versus measured airflow obstruction. Health Rep. 25, 3-11 (2014).

17. Coultas, D. B., Mapel, D., Gagnon, R. \& Lydick, E. The health impact of undiagnosed airflow obstruction in a national sample of United States adults. Am. J. Respir. Crit. Care Med. 164, 372-377 (2001).

18. Buffels, J., Degryse, J., Heyrman, J. \& Decramer, M. Office spirometry significantly improves early detection of COPD in general practice: the DIDASCO study. Chest 125, 1394-1399 (2004).

19. den Otter, J. J., van Dijk, B., van Schayck, C. P., Molema, J. \& van Weel, C. How to avoid underdiagnosed asthma/chronic obstructive pulmonary disease? J. Asthma 35, 381-387 (1998).

20. Spero, K., Bayasi, G., Beaudry, L., Barber, K. R. \& Khorfan, F. Overdiagnosis of COPD in hospitalized patients. Int. J. Chron. Obstruct. Pulmon. Dis. 12, 2417-2423 (2017).

21. Chow, C. M., Donovan, L., Manuel, D., Johansen, H. \& Tu, J. V. Regional variation in self-reported heart disease prevalence in Canada. Can. J. Cardiol. 21, 1265-1271 (2005).
22. Tu, J. V., Khan, A. M., Ng, K. \& Chu, A. Recent temporal changes in atherosclerotic cardiovascular diseases in Ontario: clinical and health systems impact. Can. J. Cardiol. 33, 378-384 (2017).

23. Dunlay, S. M. et al. Hospitalizations after heart failure diagnosis: a community perspective. J. Am. Coll. Cardiol. 54, 1695-1702 (2009).

24. Brown, A. D. et al. Hospitalization for ambulatory care-sensitive conditions: a method for comparative access and quality studies using routinely collected statistics. Can. J. Public Health 92, 155-159 (2001).

25. Kripalani, S. et al. Deficits in communication and information transfer between hospital-based and primary care physicians: implications for patient safety and continuity of care. J. Am. Med. Assoc. 297, 831-841 (2007).

26. Azad, N. \& Lemay, G. Management of chronic heart failure in the older population. J. Geriatr. Cardiol. 11, 329-337 (2014).

27. Ni, H., Nauman, D. J. \& Hershberger, R. E. Managed care and outcomes of hospitalization among elderly patients with congestive heart failure. Arch. Intern. Med. 158, 1231-1236 (1998).

28. Lainscak, M. et al. The burden of chronic obstructive pulmonary disease in patients hospitalized with heart failure. Wien. Klin. Wochenschr. 121, 309-313 (2009).

29. Ukena, C. et al. The cardiopulmonary continuum systemic inflammation as 'common soil' of heart and lung disease. Int. J. Cardiol. 145, 172-176 (2010).

30. Medical Records, Policy Statement 4-12. http://www.cpso.on.ca/PoliciesPublications/Policy/Medical-Records.(College of Physicians and Surgeons of Ontario, Toronto, 2012).

31. von Elm, E. et al. The strengthening the reporting of observational studies in epidemiology (STROBE) statement: guidelines for reporting observational studies. Int. J. Surg. 12, 1495-1499 (2014).

32. Birtwhistle, R. V. Canadian primary care sentinel surveillance network: a developing resource for family medicine and public health. Can. Fam. Physician 57, 1219-1220 (2011).

33. Canadian Institute for Health Information $(\mathrm{ClHI})$ Discharge Abstract Database (DAD) Abstracting Manual. https://secure.cihi.ca/estore/productSeries.htm? locale $=$ en\&pc $=$ PCC1252 (2017).

34. Health Information (CIHI). NACRS Manual for 2016-2017. https://secure.cihi.ca/ estore/productFamily.htm?locale $=$ en\&pf $=$ PFC3666\&media $=0$ (2016).

35. Health Quality Ontario; Ministry of Health and Long-Term Care Quality-Based Procedures: Clinical Handbook for Heart Failure (Acute and Postacute). (Health Quality Ontario, Toronto, 2015).

36. Health Quality Ontario; Ministry of Health and Long-Term Care Quality-Based Procedures: Clinical Handbook for Chronic Obstructive Pulmonary Disease (Acute and Poastacute. (Health Quality Ontario, Toronto, 2015).

37. Canadian Institute for College of Physicians and Surgeons of Nova Scotia. Guidelines for medical record keeping: council of the college of physicians and surgeons of Nova Scotia. http://cpsns.ns.ca/Portals/0/Guidelines-policies/2008medical-record.pdf (2008).

38. Williamson, T. et al. Validating the 8 CPCSSN case definitions for chronic disease surveillance in a primary care database of electronic health records. Ann. Fam. Med. 12, 367-372 (2014).

39. Lacasse, Y., Daigle, J. M., Martin, S. \& Maltais, F. Validity of chronic obstructive pulmonary disease diagnoses in a large administrative database. Can. Respir. J. 19, e5-e9 (2012).

40. Quach, S., Blais, C. \& Quan, H. Administrative data have high variation in validity for recording heart failure. Can. J. Cardiol. 26, 306-312 (2010).

41. Pampalon, R., Hamel, D., Gamache, P. \& Raymond, G. A deprivation index for health planning in Canada. Chronic Dis. Can. 29, 178-191 (2009).

42. Wilkins, R. Use of postal codes and addresses in the analysis of health data. Health Rep. 5, 157-177 (1993).

Open Access This article is licensed under a Creative Commons Attribution 4.0 International License, which permits use, sharing, adaptation, distribution and reproduction in any medium or format, as long as you give appropriate credit to the original author(s) and the source, provide a link to the Creative Commons license, and indicate if changes were made. The images or other third party material in this article are included in the article's Creative Commons license, unless indicated otherwise in a credit line to the material. If material is not included in the article's Creative Commons license and your intended use is not permitted by statutory regulation or exceeds the permitted use, you will need to obtain permission directly from the copyright holder. To view a copy of this license, visit http://creativecommons. org/licenses/by/4.0/.

(c) The Author(s) 2018 